\title{
The Effect of Intramuscular versus Intra-Amniotic Dexamethasone Administration on Blood Glucose Level in Diabetic Pregnant Women
}

\author{
Yuchun Zhu1, Xinran He1, Fang Yan², Yu Sun ${ }^{1 *}$ \\ ${ }^{1}$ Obstetrics and Gynecology Department, Peking University First Hospital, Beijing, China \\ 'Obstetrics and Gynecology Department, Cangzhou People's Hospital, Cangzhou, China \\ Email: sunyu1705@sina.com
}

Received 25 May 2016; accepted 18 June 2016; published 21 June 2016

Copyright (C) 2016 by authors and Scientific Research Publishing Inc.

This work is licensed under the Creative Commons Attribution International License (CC BY). http://creativecommons.org/licenses/by/4.0/

\section{(c) (i) Open Access}

\begin{abstract}
Background: To investigate the blood glucose level response of diabetic pregnant women when administrating antenatal dexamethasone to accelerate fetal lung maturity in different routes. Methods: This prospective cohort study included 60 pregnant women with diabetes who required promotion of fetal lung maturity in Peking University First Hospital from January 2013 to April 2015. Dexamethasone was administered by IM $(n=29)$ or IAC $(n=31)$. Blood glucose level was monitored before injection, and 1 day, 2 days and 3 days after injection. Results: In the intramuscular injection group, the preprandial and postprandial blood glucose concentration was significantly elevated in the first two days $(\mathrm{P}<\mathbf{0 . 0 0 1})$, and the postprandial blood glucose level was still higher than basal level $(P=\mathbf{0 . 0 3 4})$ on the third day. In the intra-amniotic injection group, the preprandial and postprandial blood glucose concentration was also significantly elevated on the first day $(P<0.001)$, but soon returned to the basal level on the second day. No significant difference was found in the magnitude of glucose increase between the two groups. Conclusion: Blood glucose level is significantly influenced by intramuscular injection of antenatal dexamethasone rather than amniotic cavity injection.
\end{abstract}

\section{Keywords}

Antenatal Corticosteroids, Dexamethasone, Blood glucose, Diabetes Mellitus, Pregnancy

\footnotetext{
${ }^{*}$ Corresponding author.
}

How to cite this paper: Zhu, Y.C., He, X.R., Yan, F. and Sun, Y. (2016) The Effect of Intramuscular versus Intra-Amniotic Dexamethasone Administration on Blood Glucose Level in Diabetic Pregnant Women. Open Journal of Obstetrics and Gynecology, 6, 433-437. http://dx.doi.org/10.4236/ojog.2016.67057 


\section{Introduction}

Pregnancy with diabetes is composed of pre-gestational diabetes mellitus (PGDM) and gestational diabetes mellitus (GDM). As is well known, neonatal respiratory distress syndrome (NRDS) is one of the major complications of diabetes in pregnancy, which is due to fetal lung immaturity caused by surfactant insufficiency. Promotion of fetal lung maturity is essential when spontaneous or iatrogenic preterm delivery is inevitable or when blood glucose is poorly controlled [1]. Corticosteroid is an effective approach for fetal lung maturity, which can be administered either by intramuscular injection or by amniotic cavity injection. However, it is lack of research focusing on how and to what extent the blood glucose level differs in diabetic patients through different administration routes. This study investigates the blood glucose change due to the injection of dexamethasone, in order to reveal the impact of different administration routes on diabetic pregnant women.

\section{Methods}

\subsection{Patients}

This prospective cohort study was conducted in 60 pregnant women with diabetes mellitus who required promotion of fetal lung maturity in Peking University First Hospital from Jan 2013 to Apr 2015. The diagnostic criteria for PGDM was defined as diabetes diagnosed before pregnancy or hyperglycemia before 24 gestational weeks, which involved fasting blood glucose $\geq 7 \mathrm{mmol} / \mathrm{L}$, postprandial blood glucose $\geq 11.1 \mathrm{mmol} / \mathrm{L}, \mathrm{HbA} 1 \mathrm{c} \geq 6.5 \%$, random blood glucose $\geq 11.1 \mathrm{mmol} / \mathrm{L}$ with symptoms of hyperglycemia. The $75 \mathrm{~g}$ oral glucose tolerance test was used to diagnose GDM between 24 and 28 gestational weeks, of which the threshold was $5.1 \mathrm{mmol} / \mathrm{L}, 10.0$ $\mathrm{mmol} / \mathrm{L}, 8.5 \mathrm{mmol} / \mathrm{L}$ for fasting, 1 and 2 hours respectively. A diagnosis of GDM was made when 1 or more glucose levels fall at or above the thresholds.

Fetal outcome we assessed were macrosomia, asphyxia, neonatal hypoglycemia and NRDS. Macrosomia was defined as a birth weight over $4000 \mathrm{~g}$. Asphyxia was defined as Apgar score $\leq 7$. Neonatal hypoglycemia was defined as blood glucose level less than $2.2 \mathrm{mmol} / \mathrm{L}$. A neonatologist determined the diagnosis of NRDS.

The study was approved by the Research Ethic Committees of Peking University First Hospital, and informed consents were obtained from all participated patients. The route of dexamethasone administration depended on the patient's intention. Therefore, 29 cases were enrolled in the intramuscular injection group (IM), while 31 cases were enrolled in the intra-amniotic cavity injection group (IAC). There were 7 cases of DM patients in the IM group and 13 cases in the IAC group. The rest were all GDM patients.

The patients who had multiple gestation, premature rupture of membrane, infection or medicine interfere with glucose metabolism were excluded from this study.

\subsection{Methods}

Dexamethasone was given either by intramuscular injection or by intra-amniotic cavity injection. In the IM group, patients received 4 doses of $5 \mathrm{mg}$ dexamethasone at 12 hourly-intervals. Patients in the IAC group all signed informed consent of amniocentesis when receiving one dosage of $10 \mathrm{mg}$. To avoid unexpected injury on the placenta and fetus, the procedure was performed under surveillance of ultrasonography.

One day prior to medication of dexamethasone (day 0 ), the preprandial glucose level and 2 hours postprandial level of all patients were obtained. After medication, preprandial and postprandial plasma glucose levels of 3 meals were examined on day 1 , day 2 and day 3 . Average preprandial and average postprandial glucose levels were calculated on each day. The as-prepared data were carefully analyzed according to the following procedure.

\subsection{Statistical Analysis}

Statistical analysis was performed with SAS 9.2 software for Windows. The standard deviation (Mean \pm SD) of measurement data of normal distribution was used to compare among groups with $t$ test or analysis of variance, and compared with paired $t$ test before and after treatment. Chi square test or Fisher exact probability method was used to compare the percentage of count data. All the data were examined by double tailed test and $\mathrm{P}<0.05$ was defined as statistically significant. 


\section{Results}

The general characteristics of all the participants in two groups are comparable, including age (34.76 \pm 4.06 vs. $34.10 \pm 3.26, \mathrm{P}=0.224)$, gravidity, parity, delivery weeks $(38.52 \pm 0.96$ vs. $38.32 \pm 0.77, \mathrm{P}=0.372)$ and treating weeks $(37.60 \pm 1.30$ vs. $37.86 \pm 0.98, \mathrm{P}=0.386)$. There are no complications of amniocentesis in the IAC group.

As it is shown in Table 1, the level of preprandial blood glucose has been remarkably elevated within the first 24 hours in both groups $(1.21 \pm 0.87 \mathrm{mmol} / \mathrm{L}$ vs. $1.06 \pm 1.15 \mathrm{mmol} / \mathrm{L})$ after dexamethasone injection when compared with the pre-injection level $(\mathrm{P}<0.001)$. Thereafter, on the second day, it remains elevated in the IM group $(1.52 \pm 0.82 \mathrm{mmol} / \mathrm{L}, \mathrm{P}<0.001)$ while in the IAC group it returns to pre-injection level $(\mathrm{P}=0.612)$ and continues being stable onwards. On the third day, in the IM group the preprandial level trends to go back to the pre-injection level but the difference is still of statistical significance.

The level of postprandial blood glucose has been remarkably elevated on the first day in both groups $(1.38 \pm$ $1.15 \mathrm{mmol} / \mathrm{L}$ vs. $1.34 \pm 1.26 \mathrm{mmol} / \mathrm{L})$ after dexamethasone injection when compared with the pre-injection level $(\mathrm{P}<0.001)$. Thereafter, on the second day, it remains elevated in the IM group $(1.53 \pm 0.85 \mathrm{mmol} / \mathrm{L}, \mathrm{P}<0.001)$ while in the IAC group it returns to pre-injection level $(\mathrm{P}=0.612)$. On the third day, the postprandial glucose level goes back to the D0 level in both groups.

The overall fluctuation of glucose level caused by either route of dexamethasone administration is depicted in Figure 1.

When concerning the neonatal outcome, including macrosomia, hypoglycemia, hyperbilirubinemia, asphyxia and NRDS, there are no differences between two groups (Table 2).

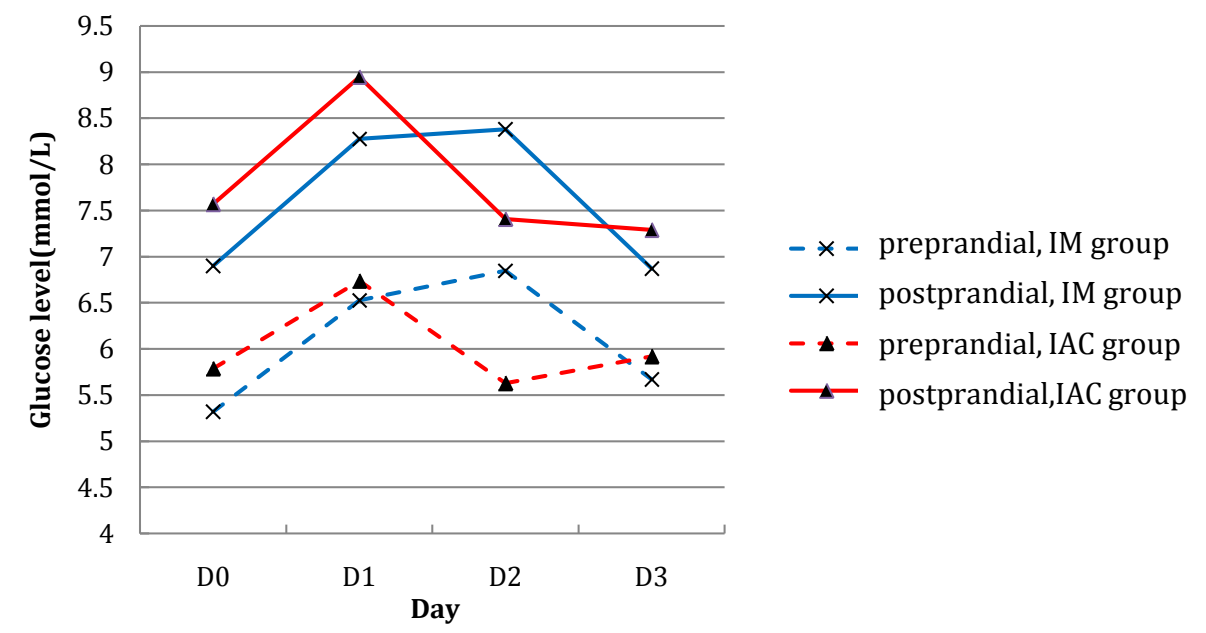

Figure 1. Curve line of glucose level fluctuation in two groups. IM: intramuscular; IAC: intraamniotic cavity.

Table 1. Preprandial and postprandial blood glucose change of two groups.

\begin{tabular}{cccc}
\hline $\begin{array}{c}\text { Glucose Level } \\
(\mathrm{mmol} / \mathrm{L})\end{array}$ & $\begin{array}{c}\text { IM Group }(\mathrm{n}=29) \\
\text { Preprandial/Postprandial }\end{array}$ & $\begin{array}{c}\text { IAC Group }(\mathrm{n}=31) \\
\text { Preprandial/Postprandial }\end{array}$ & P \\
\hline D0 & $5.32 \pm 0.70 / 6.90 \pm 0.75$ & $5.79 \pm 0.62 / 7.57 \pm 1.15$ & $0.008^{*} / 0.009^{*}$ \\
D1 & $6.53 \pm 0.76 / 8.28 \pm 1.20$ & $6.74 \pm 1.29 / 8.95 \pm 1.66$ & $0.467 / 0.091$ \\
P\# & $<0.001^{*} /<0.001^{*}$ & $<0.001^{*} /<0.001^{*}$ & $<0.001^{*} / 0.010^{*}$ \\
D2 & $6.85 \pm 0.90 / 8.38 \pm 0.93$ & $5.63 \pm 0.81 / 7.41 \pm 1.41$ & $0.657 / 0.506$ \\
P\# & $<0.001^{*} /<0.001^{*}$ & $0.612 / 0.698$ & $5.92 \pm 2.04 / 7.29 \pm 2.34$ \\
D3 & $5.67 \pm 0.44 / 6.87 \pm 0.55$ & $0.826 / 0.825$ & \\
P\# & $0.034^{*} / 0.327$ & . & \\
\hline
\end{tabular}

${ }^{*} \mathrm{P}<0.05$ is defined as statistically significant. \#Compared with D0. IM: intramuscular; IAC: intra-amniotic cavity; D0: pre-injection; D1: the first day after injection; D2: the second day after injection; D3: the third day after injection. 
Table 2. Neonatal outcomes.

\begin{tabular}{cccc}
\hline & IM Group $(\mathrm{n}=28)$ & IAC Group $(\mathrm{n}=26)$ & P \\
\hline Macrosomia & $4(14.29 \%)$ & $3(11.54 \%)$ & 0.162 \\
Asphyxia & $1(3.57 \%)$ & $0(0.00 \%)$ & 0.402 \\
Hypoglycemia & $4(14.29 \%)$ & $1(3.85 \%)$ & 0.087 \\
NRDS & $0(0.00 \%)$ & $0(0.00 \%)$ & 1.000 \\
Hyperbilirubinemia & $4(14.29 \%)$ & $7(26.92 \%)$ & 0.076 \\
\hline
\end{tabular}

IM: intramuscular; IAC: intra-amniotic cavity; NRDS: neonatal respiratory distress.

\section{Discussion}

It is verified by abundant large clinical trials that antenatal corticosteroid therapy is indicated for women who are at high risk of premature delivery as its effectiveness for fetal lung maturation, especially in the cases when pregnancies complicates with diabetes since fetal lung maturity is delayed in such patients. The ACOG (American College of Obstetricians and Gynecologists) recommends adoption of glucocorticoids for pregnant women who are at risk of preterm delivery within 7 days before 34 weeks [1]. Despite of this, there is no definite conclusion whether glucocorticoids exert great impact on the blood glucose level in diabetic pregnancies. It is reported that the glucose level is witnessed to be prominently elevated after given glucocorticoids intramuscularly, especially in those pregnant women with diabetes [2] [3]. Meanwhile, whether accelerating fetal lung maturation by amniotic cavity injection impacts blood glucose dramatically or not is not well investigated. Up till now, related research documentation has been mainly limited to animal research beyond China. In China, some researchers are concerning the side effects of glucocorticoids that are used for accelerating fetal lung maturity. This study focuses on the blood glucose variation via different routes of medication in diabetic pregnancies.

Our study shows the blood glucose level has increased during 48 hours since intramuscular dexamethasone injection $(\mathrm{P}<0.001)$, and the postprandial blood glucose is still higher than basal level on the third day $(\mathrm{P}=$ 0.034). Comparatively, in the IAC group, the glucose level rises and falls relatively evenly, which peaks on the first day $(\mathrm{P}<0.001)$, but soon returns to the basal level on the second day. Based on this result, we deduce that intra-amniotic administration exerts less impact on the glucose fluctuation in diabetic pregnant women.

It is well known that antenatal glucocorticoids induce protein synthesis in surfactant-producing type II cells in the fetal lung, which prevents neonatal respiratory distress and lowers mortality of preterm babies. However, maternal corticosteroid administration firstly causes maternal reaction including hyperglycemia before its therapeutic effect on the fetus via the placenta. In contrast, corticosteroids in the amniotic fluid are directly absorbed by fetal gastrointestinal epithelium or by fetal respiration through intra-amniotic route, independent of maternal circulation. Thus, such dose of corticosteroids does not lead to maternal immune-suppression and glucose metabolism interruption.

Furthermore, the surfactant-producing effect of glucocorticoids is blunted by insulin in diabetic pregnant women. Maternal intramuscular glucocorticoids result in maternal hyperglycemia, which leads to fetal hyperglycemia. Fetal pancreatic beta cells secrete more insulin in response, which attenuates the effect of glucocorticoids consequently. Intra-amniotic administration of betamethasone has been reported to be superior to maternal intramuscular administration in terms of fetal surfactant induction in monkey models [4]. However, there are controversies around the community. One study indicated less effective results with more risks of fetal death and fetal lung injury from intra-amniotic betamethasone in goat models [5].

From the neonatal aspect, if fetal hyperinsulinemia occurred, the neonate would be prone to have hypoglycemia due to the shutdown of glucose supply from the mother. Severe hypoglycemia can be hazardous to the developing brain. Transient extreme hyperglycemia is detrimental to both mothers and babies. Extra maternal monitoring and treatment with insulin have to be considered. Based on our study results, we infer that the optimal timing of delivery after intramuscular administration of corticosteroids would probably be beyond $48 \mathrm{hrs}$ if glycemia is not well controlled in order to avoid neonatal hypoglycemia. The NICE (National Institute for Health and Care Excellence) guideline recommends giving additional insulin to diabetic women receiving steroids [6]. In one report from Mexico [7], a requirement of extra amount of insulin depending on the type and 
severity of diabetes mellitus was documented after antenatal betamethasone therapy. The greatest changes occurred between days 2 to 4 after betamethasone. In the review by Kalra [8], insulin dose adjustment and fetal-maternal surveillance were suggested after antenatal corticosteroid therapy.

Nevertheless, it is of vital importance to maintain euglycemia in diabetic pregnancies, so that we can avoid or reduce the administration of corticosteroids bringing out fewer side effects. However if it is necessary to accelerate fetal lung maturity, intra-amniotic route is probably superior to intramuscular route in considerations of efficacy and side effects in diabetic pregnancies. And intra-amniotic route also facilitates fetal lung maturity test. Meanwhile, since amniocentesis is an invasive procedure, the risk and benefits should be balanced and patient's request should be considered when making the decision.

Finally, the sample size is relatively limited in our research. Selection bias might be another drawback of this study. Despite this, further studies should be conducted to investigate the optimal route to accelerate fetal lung maturity in diabetic pregnancies.

\section{Acknowledgements}

This study is supported by the personnel training project of Peking University-Tsinghua University Joint Center for Life Sciences (TPCLS).

\section{References}

[1] ACOG Committee on Obstetric Practice, ACOG Committee Opinion No. 475 (2011) Antenatal Corticosteroid Therapy for Fetal Maturation. Obstetrics \& Gynecology, 117, 422-424. http://dx.doi.org/10.1097/AOG.0b013e31820eee00

[2] Refuerzo, J.S., Garg, A., Rech, B., Ramin, S.M., Vidaeff, A. and Blackwell, S.C. (2012) Continuous Glucose Monitoring in Diabetic Women Following Antenatal Corticosteroid Therapy: A Pilot Study. American Journal of Perinatology, 29, 335-338. http://dx.doi.org/10.1055/s-0031-1295642

[3] Kreiner, A., Gil, K. and Lavin, J. (2012) The Effect of Antenatal Corticosteroids on Maternal Serum Glucose in Women with Diabetes. Open Journal of Obstetrics and Gynecology, 2, 112-115. http://dx.doi.org/10.4236/ojog.2012.22021

[4] Gilbert, W.M., Eby-Wilkens, E., Plopper, C., Whitsett, J.A. and Tarantal, A.F. (2001) Fetal Monkey Surfactants after Intra-Amniotic or Maternal Administration of Betamethasone and Thyroid Hormone. Obstetrics \& Gynecology, 98, 466-470. http://dx.doi.org/10.1097/00006250-200109000-00019

[5] Sezik, M., Antalyali, M., Ozmen, O., Haligur, M., Koker, A., Ozkaya, O., et al. (2012) Ultra-High Dose of Intra-Amniotic or Direct Fetal Intramuscular Betamethasone for Lung Maturation in the Preterm Goat Model. European Journal of Obstetrics \& Gynecology and Reproductive Biology, 164, 15-23. http://dx.doi.org/10.1016/j.ejogrb.2012.05.031

[6] National Institute for Health and Clinical Excellence (2014) Diabetes in Pregnancy: Management of Diabetes and Its Complications from Pre-Conception to the Postnatal Period. London: NICE, 2008, Accessed October 15.

[7] Ramírez-Torres, M.A., Pérez-Monter, S.E., Espino y Sosa, S. and Ibargüengoitia-Ochoa, F. (2011) Effect of Betamethasone in Blood Glucose Levels in Pregnant Diabetic Women at Risk of Preterm Birth. Ginecología y Obstetricia de México, 79, 565-571.

[8] Kalra, S., Kalra, B. and Gupta, Y. (2014) Glycemic Management after Antenatal Corticosteroid Therapy. North American Journal of Medical Sciences, 6, 71-76. http://dx.doi.org/10.4103/1947-2714.127744 